PAPER

\title{
Driver landmark and traffic sign identification in early Alzheimer's disease
}

\author{
E Y Uc, M Rizzo, S W Anderson, Q Shi, J D Dawson
} See end of article for
authors' affiliations

....................

Correspondence to: Dr Ergun Y Uc, Department of Neurology, University of lowa Hospitals and Clinics, 200 Hawkins Drive, lowa City, IA 52242, USA; ergun-uc@uiowa.edu

Received 7 July 2004 In revised form 5 September 2004 Accepted 10 October 2004
J Neurol Neurosurg Psychiatry 2005;76:764-768. doi: 10.1136/jnnp.2004.049338

Objective: To assess visual search and recognition of roadside targets and safety errors during a landmark and traffic sign identification task in drivers with Alzheimer's disease.

Methods: 33 drivers with probable Alzheimer's disease of mild severity and 137 neurologically normal older adults underwent a battery of visual and cognitive tests and were asked to report detection of specific landmarks and traffic signs along a segment of an experimental drive.

Results: The drivers with mild Alzheimer's disease identified significantly fewer landmarks and traffic signs and made more at-fault safety errors during the task than control subjects. Roadside target identification performance and safety errors were predicted by scores on standardised tests of visual and cognitive function.

Conclusions: Drivers with Alzheimer's disease are impaired in a task of visual search and recognition of roadside targets; the demands of these targets on visual perception, attention, executive functions, and memory probably increase the cognitive load, worsening driving safety.
S afe automobile driving requires a driver to perform multiple competing tasks and attend to a host of objects and ongoing events, while simultaneously monitoring traffic with central and peripheral vision to avoid roadway hazards. ${ }^{1}$ Impairments of visual acuity and visual fields increase crashes and traffic violations. ${ }^{2}$ However, drivers with certain neurological conditions may fail to perceive critical roadside targets and dangers even in the absence of a measurable field defect on standard perimetry or diminished visual acuity. ${ }^{1}$

Alzheimer's disease affects processing of visual sensory cues and may produce attentional decline and agnosia. ${ }^{3-12}$ These deficits can impair drivers' processing of visual information such as roadway landmarks and traffic signs that provide key information about a driver's route, upcoming road hazards, and safety regulations. To address this real world problem, we used an instrumented vehicle (IV) to test the hypothesis that drivers with early Alzheimer's disease have impairments on a landmark and traffic sign identification task (LTIT). We also tested whether the impaired drivers would commit more safety errors under the influence of the cognitive load imposed by the LTIT, placing them at greater risk of a crash. Finally, we tested whether LTIT performance and safety errors could be predicted by visual and cognitive measures sensitive to decline in early Alzheimer's disease.

\section{METHODS \\ Subjects}

Subjects were 33 participants with mild Alzheimer's disease and 137 neurologically normal controls. Both groups had comparable years of education, but the Alzheimer subjects were older (table 1). The male predominance in the Alzheimer group may reflect the fact that women in this age group had not traditionally been the main driver in the family and more readily relinquished their driving privileges once they developed Alzheimer's disease. Subjects with neurological disorders other than Alzheimer's disease were excluded. No subject had acute, confounding medical or psychiatric conditions. Participants with Alzheimer's disease were recruited from a registry in the department of neurology. All Alzheimer patients had objective evidence of memory impairment and related cognitive complaints that interfered with their social or occupational life consistent with the NINCDS-ADRDA criteria. ${ }^{13}$ Mini-mental state examination (MMSE) screening in patients with Alzheimer's disease (mean (SD) score, 26.1 (3.0)) reflected mild early cognitive decline. Impairments on memory and other cognitive domains on a standardised battery of neuropsychological tests (table 1) were consistent with early Alzheimer's disease. ${ }^{14}$ Computed tomographic and magnetic resonance imaging scans of the brain excluded destructive lesions caused by cerebrovascular and neoplastic disease.

Control participants were recruited from volunteers in the local community. All participants held a current valid state driver's license and were still driving, although some had reduced driving activity because of self imposed or family imposed restrictions.

The institutional review board approved the project and informed consent was obtained in accord with institutional and federal guidelines.

\section{Cognitive and visual battery}

All participants were tested on a battery of cognitive and visual tasks (tables 1 and 2). Judgment of Line Orientation (JLO) assessed visuospatial perception. Visuoconstructional ability was tested using the Rey-Osterreith Complex Figure Test copy version (CFT-copy) and the block design subtest (Blocks) from the WAIS-R (Wechsler Adult Intelligence Scale, revised). The CFT-recall version and the Benton Visual Retention Test (BVRT) tested non-verbal memory, while the Rey Auditory Verbal Learning Test (AVLT) provided an index

Abbreviations: ARGOS, Automobile for Research in Ergonomics and Safety; AVLT-recall, auditory verbal learning test-recall; Blocks, block design subtest; BVRT, Benton visual retention test; CFT-copy, complex figure test-copy; CFT-recall, complex figure test-recall; Cogstat, composite measure of cognitive impairment; COWA, controlled oral word association; CS, contrast sensitivity; FVA, far visual acuity; JLO, judgment of line orientation; LTIT, landmark and traffic sign identification task; MMSE, mini-mental state examination; NINCDS-ADRDA, National Institute of Neurological and Communicative Disorders and StrokeAlzheimer's Disease and Related Disorders Association; NVA, near visual acuity; SFM, structure from motion; TMT-B, trail making test subtest B; UFOVTOT, total of subtests of the useful field of view task. 
Table 1 Comparison of Alzheimer's disease and control groups

\begin{tabular}{|c|c|c|c|c|}
\hline \multirow[b]{2}{*}{ Variable } & \multirow[b]{2}{*}{$A D(n=33)$} & \multirow[b]{2}{*}{ Normal $(n=137)$} & \multicolumn{2}{|l|}{ p Values } \\
\hline & & & Crude & Age adjusted \\
\hline \multicolumn{5}{|l|}{ Demographics } \\
\hline $\operatorname{Sex}(M, F)$ & 28,5 & 69,68 & & \\
\hline Age (y) & $76.1(6.3)$ & $64.3(11.4)$ & $<0.0001$ & \\
\hline Education (y) & $15.2(3.2)$ & $15.6(2.5)$ & 0.3545 & \\
\hline Symptom duration (y) & $2.1(1.5)$ & & & \\
\hline \multicolumn{5}{|l|}{ Cognitive tests } \\
\hline Cogstat & $298(47)$ & $405(45)$ & $<0.0001$ & $<0.0001$ \\
\hline AVLT-recall & $3.2(2.5)$ & $10.5(3.0)$ & $<0.0001$ & $<0.0001$ \\
\hline BVRT (errors) & $9.2(3.2)$ & $4.4(2.5)$ & $<0.0001$ & $<0.0001$ \\
\hline CFT-recall & $9.1(4.5)$ & $15.8(5.7)$ & $<0.0001$ & $<0.0001$ \\
\hline JLO & $23.5(4.1)$ & $26.2(3.6)$ & 0.0005 & 0.0156 \\
\hline Blocks & $23.5(10.0)$ & $40.4(10.8)$ & $<0.0001$ & $<0.0001$ \\
\hline CFT-copy & $28.7(4.3)$ & $32.0(3.3)$ & $<0.0001$ & 0.0002 \\
\hline TMT-B & $158(74)$ & 79 (39) & $<0.0001$ & $<0.0001$ \\
\hline COWA & $34.2(10.8)$ & $39.3(10.8)$ & 0.0160 & 0.1021 \\
\hline \multicolumn{5}{|l|}{ Visual tests } \\
\hline NVA & $0.057(0.068)$ & $0.018(0.037)$ & $<0.0001$ & 0.0011 \\
\hline FVA & $0.030(0.113)$ & $-0.098(0.116)$ & $<0.0001$ & 0.0013 \\
\hline CS & $1.64(0.17)$ & $1.85(0.14)$ & $<0.0001$ & $<0.0001$ \\
\hline SFM & 12.7 (7.3) & $10.3(2.8)$ & 0.1235 & 0.0067 \\
\hline UFOVTOT (ms) & 1520 (1537) & $615(236)$ & $<0.0001$ & $<0.0001$ \\
\hline \multicolumn{5}{|c|}{$\begin{array}{l}\text { Values are mean (SD) or } \mathrm{n} \text {. } \\
\text { AD, Alzheimer's disease; AVLT-recall, auditory verbal learning test-recall; Blocks, block design subtest; BVRT, } \\
\text { Benton visual retention test; CFT-copy, complex figure test-copy; CFT-recall, complex figure test-recall; Cogstat, } \\
\text { composite measure of cognitive impairment; COWA, controlled oral word association; CS, contrast sensitivity; } F \text {, } \\
\text { female; FVA, far visual acuity; JLO, judgment of line orientation; M, male; NVA, near visual acuity; SFM, structure } \\
\text { from motion; TMT-B, trail making test subtest B; UFOVTOT, total of subtests of the useful field of view task; y, years. }\end{array}$} \\
\hline
\end{tabular}

of anterograde verbal memory. The Trail Making Test subtest B (TMT-B) and Controlled Oral Word Association (COWA) were used to test various aspects of executive function. These tasks are described in detail elsewhere. ${ }^{15}$ We calculated a composite measure of cognitive impairment (Cogstat) by assigning standard $\mathrm{T}$ scores (mean $=50, \mathrm{SD}=10$ ) to each of the eight tests from the neuropsychological assessment battery. ${ }^{5}$

The Useful Field of View (UFOV) task (Visual Attention Analyser, Visual Resources Inc), measures speed (in ms) of visual processing, divided attention, and selective attention. ${ }^{16}$ We used the sum of four subtests of the UFOV task (UFOVTOT) in our analyses. UFOV loss has predicted crashes in the state records of drivers with visual and cognitive impairments. ${ }^{17}{ }^{18}$ Contrast sensitivity (CS) was assessed using the Pelli-Robson chart. ${ }^{19}$ The best corrected visual acuity was measured using the ETDRS chart ${ }^{20}$ for far visual acuity (FVA) and the reduced Snellen chart for near visual acuity (NVA), both expressed as LogMAR (logarithm of the minimum angle of resolution), with 0 representing 20/20 vision. Perception of three dimensional structure-from-motion (SFM) and of motion direction were tested using computer generated animation sequences. ${ }^{7}$

\section{Experimental drive}

The experimental drive was conducted aboard a dual control instrumented vehicle known as ARGOS (the Automobile for Research in Ergonomics and Safety), a mid-sized 1995 Ford Taurus station wagon with automatic transmission and with hidden instrumentation and sensors. ${ }^{21} 22$ Data on steering wheel position, normalised accelerator and brake pedal position, lateral and longitudinal acceleration, and vehicle speed were obtained at $10 \mathrm{~Hz}$. Driver's lane tracking and visual scanning activity of the environment were videotaped at 30 frames per second.

The experimenter sat in the front passenger seat to score the on-road performance and operate the dual controls in case of emergency. The experimental drive started after the driver acclimated to ARGOS and was carried out only during daylight and in good weather. It lasted approximately 45 minutes, and consisted of "on task" (for example, while performing LTIT) and "no task" segments.

Table 2 Comparison of Alzheimer's disease and control groups using one to one age matching

\begin{tabular}{|c|c|c|c|}
\hline Variable & $A D(n=23)$ & Normal $(n=23)$ & p Value \\
\hline \multicolumn{4}{|l|}{ Demographics } \\
\hline Age $(y)$ & $74.1(6.1)$ & $74.1(6.1)$ & 1.0000 \\
\hline Education (y) & $15.2(3.0)$ & $15.7(2.2)$ & 0.1515 \\
\hline Symptom duration (y) & $2.0(1.6)$ & & \\
\hline \multicolumn{4}{|l|}{ Cognitive tests } \\
\hline Cogstat & $294(47)$ & $397(43)$ & $<0.0001$ \\
\hline AVLT-recall & $3.0(2.5)$ & $10.3(2.8)$ & $<0.0001$ \\
\hline BVRT (errors) & $9.4(3.2)$ & $5.1(2.2)$ & $<0.0001$ \\
\hline CFT-recall & $9.3(4.7)$ & $15.7(5.1)$ & 0.0005 \\
\hline JLO & $23.0(4.4)$ & $25.1(4.5)$ & 0.2242 \\
\hline Blocks & $23.4(8.9)$ & $38.8(10.6)$ & $<0.0001$ \\
\hline CFT-copy & $29.1(4.0)$ & 31.7 (3.5) & 0.0508 \\
\hline TMT-B & $154(81)$ & $82(27)$ & $<0.0001$ \\
\hline COWA & $33.2(11.6)$ & $41.11(9.4)$ & 0.0002 \\
\hline \multicolumn{4}{|l|}{ Visual tests } \\
\hline NVA & $0.056(0.070)$ & $0.022(0.036)$ & 0.0179 \\
\hline FVA & $0.034(0.124)$ & $-0.043(0.129)$ & 0.0324 \\
\hline CS & $1.66(0.18)$ & $1.79(0.16)$ & 0.0057 \\
\hline SFM & $14.0(8.3)$ & $10.4(2.5)$ & 0.0723 \\
\hline UFOVTOT (ms) & $1257(231)$ & 778 (194) & $<0.0001$ \\
\hline
\end{tabular}

Values are mean (SD).

$A D$, Alzheimer's disease; AVLT-recall, auditory verbal learning testrecall; Blocks, block design subtest; BVRT, Benton visual retention test; CFT-copy, complex figure test-copy; CFT-recall, complex figure testrecall; Cogstat, composite measure of cognitive impairment; COWA, controlled oral word association; CS, contrast sensitivity; FVA, far visual acuity; JLO, judgment of line orientation; NVA, near visual acuity; SFM, structure from motion; TMT-B, trail making test subtest $B$; UFOVTOT, total of subtests of the useful field of view task; $y$, years. 


\section{Landmark and traffic sign identification task}

LTIT was administered as part of a sequence of on-the-road tasks in ARGOS. Drivers were asked to look for and report verbally on traffic signs and restaurants (a ubiquitous type of roadside landmark) along a one mile commercial segment of a four lane divided highway approximately one minute before these stimuli started to appear. These targets were classified as high saliency or low saliency stimuli, based on ratings and detection rates of drivers tested in pilot studies. For example, a speed limit sign is a high saliency stimulus detected by nearly all subjects, while a small and low mile marker is of lower saliency and is missed by some normal subjects. Likewise, a restaurant situated right on the road in its own building is a high saliency stimulus, whereas a delicatessen within a grocery store, or a restaurant where the sign or building can be seen from afar but is not on the route, is considered a lower saliency stimulus. There were in all 16 road signs (11 high saliency) and 13 restaurants (six high saliency) along the route. Dependent measures were the percentage of landmarks and traffic signs identified and the number of at-fault safety errors such as erratic steering, lane deviation, shoulder incursion, stopping or slowing in unsafe circumstances, and unsafe intersection behaviour during the task.

\section{Statistical analysis}

We compared the Alzheimer's disease and control groups with respect to demographic, visual, cognitive, and LTIT outcome measures using the Wilcoxon rank sum test. We used regression models to adjust the LTIT outcome comparisons for age, sex, visual acuity, and previous familiarity with the route. Because of the significant age difference between the groups, we also compared the LTIT outcomes, cognitive tests, and vision tests using one to one age matching.

We used Fisher's exact test to compare the proportion of drivers in each group who made at-fault safety errors. We compared demographic factors and cognitive and vision test scores of drivers with Alzheimer's disease who drove without committing any safety errors with those who made safety errors.

We calculated Spearman correlation coefficients for the LTIT outcome measures with the cognitive and visual tests. We used stepwise linear regression to identify predictors of total landmark and traffic sign identification percentages, and ordinal logistic regression for at-fault safety errors. First,
Table 4 Outcome measures of the landmark and traffic sign identification task in Alzheimer's disease and normal controls with one to one age matching, compared using the Wilcoxon rank sum test

\begin{tabular}{|c|c|c|c|}
\hline & $A D(n=23)$ & $\begin{array}{l}\text { Normal } \\
(n=23)\end{array}$ & p Value \\
\hline \multicolumn{4}{|l|}{ Landmark identification (\%) } \\
\hline All & $29.1(14.7)$ & 44.5 (14.8) & 0.0056 \\
\hline High saliency only & $46.4(22.4)$ & $68.1(21.3)$ & 0.0002 \\
\hline \multicolumn{4}{|l|}{ Traffic sign identification (\%) } \\
\hline All & $44.9(25.0)$ & $68.5(20.1)$ & 0.0013 \\
\hline High saliency only & $54.7(27.6)$ & $80.9(21.5)$ & 0.0002 \\
\hline \multicolumn{4}{|l|}{ Total LTIT identification (\%) } \\
\hline All & $37.7(13.8)$ & $57.7(16.4)$ & 0.0001 \\
\hline High saliency only & $51.9(19.4)$ & $76.7(20.0)$ & 0.0001 \\
\hline \multicolumn{4}{|l|}{ At-fault safety errors } \\
\hline During LTIT & $1.8(1.7)$ & $0.5(1.0)$ & 0.0009 \\
\hline Baseline & $5.9(5.3)$ & $2.0(1.9)$ & 0.0058 \\
\hline
\end{tabular}

Values are mean (SD).

AD, Alzheimer's disease; LTIT, landmark and traffic sign identification task.

the individual components of Cogstat were used to model for cognitive predictors, followed by modelling for visual variables that was adjusted for general cognitive performance by using Cogstat in the model. This allowed us to see the overall effect of the main two classes of variables (cognitive and visual).

\section{RESULTS}

Drivers with Alzheimer's disease identified a significantly smaller percentage of restaurants and traffic signs, and committed more at-fault safety errors during LTIT, than the neurologically normal controls (table 3 ). The difference between the groups persisted after adjusting for familiarity with the neighbourhood, age, far and near visual acuity, and sex. The Alzheimer group also committed more at-fault safety errors at baseline segments of the drive (that is, when no secondary task like LTIT was administered) (tables 3 and 4). Drivers with Alzheimer's disease continued to show a greater number of safety errors than the controls, even after adjusting the errors during LTIT for baseline errors

Table 3 Outcome measures of the LTIT in Alzheimer's disease $(n=33)$ and normal controls $(n=137)$, compared using Wilcoxon rank sum test

\begin{tabular}{|c|c|c|c|c|c|c|c|c|c|c|}
\hline & \multirow[b]{2}{*}{$A D$} & \multirow[b]{2}{*}{ Normal } & \multirow{2}{*}{$\begin{array}{l}\text { Crude } \\
\text { p value }\end{array}$} & \multicolumn{7}{|c|}{ Adjusted $p$ values } \\
\hline & & & & Age & NVA & FVA & CS & Famil & Sex & ALL $^{*}$ \\
\hline \multicolumn{11}{|l|}{ Landmark identification (\%) } \\
\hline All & $28.9(15.5)$ & $45.4(15.7)$ & $<0.0001$ & 0.0001 & $<0.0001$ & $<0.0001$ & 0.0030 & $<0.0001$ & $<0.0001$ & 0.0320 \\
\hline High saliency only & $44.9(22.6)$ & $67.9(20.0)$ & $<0.0001$ & $<0.0001$ & $<0.0001$ & $<0.0001$ & 0.0011 & $<0.0001$ & $<0.0001$ & 0.0071 \\
\hline \multicolumn{11}{|l|}{ Traffic sign identification (\%) } \\
\hline All & $44.4(22.9)$ & $72.0(17.1)$ & $<0.0001$ & $<0.0001$ & $<0.0001$ & $<0.0001$ & $<0.0001$ & $<0.0001$ & $<0.0001$ & $<0.0001$ \\
\hline High saliency only & $53.8(26.2)$ & $83.9(17.2)$ & $<0.0001$ & $<0.0001$ & $<0.0001$ & $<0.0001$ & $<0.0001$ & $<0.0001$ & $<0.0001$ & $<0.0001$ \\
\hline \multicolumn{11}{|l|}{ Total LTIT identification (\%) } \\
\hline All & $37.4(13.1)$ & $60.0(13.2)$ & $<0.0001$ & $<0.0001$ & $<0.0001$ & $<0.0001$ & $<0.0001$ & $<0.0001$ & $<0.0001$ & $<0.0001$ \\
\hline High saliency only & $50.8(18.6)$ & $78.5(14.7)$ & $<0.0001$ & $<0.0001$ & $<0.0001$ & $<0.0001$ & $<0.0001$ & $<0.0001$ & $<0.0001$ & $<0.0001$ \\
\hline \multicolumn{11}{|l|}{ At-fault safety errors } \\
\hline During LTIT & $1.8(1.6)$ & $0.4(0.8)$ & $<0.0001$ & $<0.0001$ & $<0.0001$ & $<0.0001$ & $<0.0001$ & $<0.0001$ & $<0.0001$ & $<0.0001$ \\
\hline Baseline & $5.6(5.1)$ & $1.7(2.1)$ & $<0.0001$ & $<0.0001$ & $<0.0001$ & $<0.0001$ & $<0.0001$ & $<0.0001$ & $<0.0001$ & $<0.0001$ \\
\hline
\end{tabular}

Values are mean (SD).

*Adjustment for all covariates together.

$A D$, Alzheimer's disease; CS, contrast sensitivity; Famil, familiarity; FVA, far visual acuity; LTIT, landmark and traffic sign identification task; NVA, near visual acuity. 
$(p<0.0001)$. However, on a straight segment of the drive with no task load, there was no difference between the groups in basic vehicular control measured by the standard deviation of steering wheel position (degrees), number of large changes ( $>6$ degrees) in steering wheel position/min, and the standard deviation of mean speed (data available upon request).

The proportion of subjects who made at least one at-fault safety error was higher in the Alzheimer group (table 5) $(p<0.0001)$. The difference persisted even when the cut off value of at least two errors was used (table 5) $(\mathrm{p}<0.0001)$. There were no significant differences in age, MMSE score, or cognitive and vision tests between the "safe" and the "unsafe" subgroups of Alzheimer's disease subjects (Wilcoxon rank sum test). The only exception was that the "unsafe" group performed worse on Cogstat $(p=0.0375)$, AVLT-recall $(p=0.0351)$, and CS $(p=0.0461)$ when the cut off value of at least two errors was used. There was no difference in LTIT outcomes between the subgroups in either comparison.

To further assess the effect of age difference between the two experimental groups, we also analysed the LTIT outcome measures and cognitive/visual tests using a one to one age matched control group which included 23 perfectly matching pairs. This additional analysis produced very similar results to those obtained from the analyses in the whole experimental group. Specifically, LTIT main outcome measures (table 4) and critical visual and cognition test scores did not differ between these tightly matched experimental groups except for COWA and SFM (table 2). These findings supported our use of all the data from the whole experimental group (33 Alzheimer subjects and 137 controls), which provided increased statistical power for modelling of independent predictors of LTIT outcomes.

The Alzheimer group performed worse than the control group on several neuropsychological and visual tests showing mild dementia and visual perception and processing deficits (table 1). Measures of verbal (AVLT-recall) and non-verbal memory (CFT-recall, BVRT), executive function (TMT-B, COWA), visual perception (FVA, NVA, CS), visual attention (UFOVTOT), visuoconstructional abilities (CFT-copy, Blocks, JLO), and overall cognitive function (Cogstat) correlated significantly (Spearman coefficients) with the outcome measures of the LTIT (table 6). Using regression analyses as described above, we identified TMT-B $(p<0.0001)$, AVLTrecall $(p<0.0001), C S(p=0.0007)$, and JLO $(p=0.0379)$ as important predictors of total landmark and traffic sign identification percentage for all targets (high and low saliency together). These same four tests emerged also as predictors of identification rate of the high saliency target subset. AVLT-recall $(p=0.0018)$ and SFM $(p=0.0299)$ were predictors of at-fault safety errors.
Table 6 Significant Spearman correlation coefficients for outcome measures of the LTIT

\begin{tabular}{llrl}
\hline & Variable & \multicolumn{1}{l}{$\boldsymbol{R}_{\mathbf{s}}$} & p Value \\
\hline Total LTIT identification (\%) & UFOVTOT & -0.52 & $<0.0001$ \\
(all targets) & AVLT-recall & 0.50 & $<0.0001$ \\
& Cogstat & 0.48 & $<0.0001$ \\
& TMT-B & -0.45 & $<0.0001$ \\
CS & 0.44 & $<0.0001$ \\
& Blocks & 0.40 & $<0.0001$ \\
FVA & -0.40 & $<0.0001$ \\
& BVRT & -0.38 & $<0.0001$ \\
JLO & 0.25 & 0.0009 \\
& CFT-copy & 0.23 & 0.0023 \\
& COWA & 0.21 & 0.0053 \\
CFT-recall & 0.21 & 0.0070 \\
& NVA & -0.20 & 0.0086 \\
& & & \\
At-fault safety errors & AVLT-recall & -0.28 & 0.0002 \\
& BVRT & 0.27 & 0.0021 \\
& Blocks & -0.24 & 0.0022 \\
& CS & -0.23 & 0.0022 \\
& Cogstat & -0.23 & 0.0035 \\
& UFOVTOT & 0.22 & 0.0037 \\
& CFT-recall & -0.18 & 0.0194 \\
CFT-copy & -0.17 & 0.0263 \\
& FVA & 0.16 & 0.0344 \\
TMT-B & 0.16 & 0.0408 \\
& & &
\end{tabular}

AVLT-recall, auditory verbal learning test-recall; Blocks, block design subtest; BVRT, Benton visual retention test; CFT-copy, complex figure testcopy; CFT-recall, complex figure test-recall; Cogstat, composite measure of cognitive impairment; COWA, controlled oral word association; CS, contrast sensitivity; FVA, far visual acuity; JLO, judgment of line orientation; LTIT, landmark and traffic sign identification task; NVA, near visual acuity; SFM, structure from motion; TMT-B, trail making test subtest $B$; UFOVTOT, total of subtests of the useful field of view task.

\section{DISCUSSION}

The findings in this study support our hypothesis that drivers with Alzheimer's disease perform worse than neurologically normal drivers on an LTIT (tables 3 and 4). These group differences could not be explained by the older age or predominantly male sex of the Alzheimer subjects. Performance on standardised tests of visual perception, processing, attention, verbal and visual memory, executive functions, and visuoconstructional abilities correlated with the LTIT outcome measures (table 5) consistent with its demands on vision and cognition. These demands probably increased the cognitive load during driving, which may explain the larger number of at-fault safety errors we measured in these drivers, who have limited cognitive resources. This view is supported by the persistence of the significant between-group difference in at-fault safety errors during the LTIT, even after adjusting for baseline errors, and the lack of a group difference in basic vehicular control during a straight away baseline segment.

Table 5 Classification of subjects based on their driving safety

\begin{tabular}{|c|c|c|c|c|c|c|c|}
\hline \multirow[b]{2}{*}{ At-fault safety errors } & \multicolumn{4}{|l|}{ AD } & \multicolumn{2}{|c|}{ Normal } & \multirow[b]{2}{*}{ p Value } \\
\hline & $\mathrm{n}$ & $\%$ & Age (y) & MMSE & $\mathrm{n}$ & $\%$ & \\
\hline $\begin{array}{l}\text { Classification } 1 \\
1 \text { or more } \\
\text { None }\end{array}$ & $\begin{array}{r}25 \\
8\end{array}$ & $\begin{array}{l}(75.8 \%) \\
(24.2 \%)\end{array}$ & $\begin{array}{l}76.3(6.5) \\
75.6(6.1)\end{array}$ & $\begin{array}{l}25.9(3.1) \\
26.8(2.9)\end{array}$ & $\begin{array}{l}41 \\
96\end{array}$ & $\begin{array}{l}(29.9 \%) \\
(70.1 \%)\end{array}$ & $<0.0001$ \\
\hline $\begin{array}{l}\text { Classification } 2 \\
2 \text { or more } \\
\text { None or } 1\end{array}$ & $\begin{array}{l}17 \\
16\end{array}$ & $\begin{array}{l}(51.5 \%) \\
(48.5 \%)\end{array}$ & $\begin{array}{l}77.9(3.6) \\
74.3(9.2)\end{array}$ & $\begin{array}{l}26.5(2.4) \\
25.7(3.5)\end{array}$ & $\begin{array}{r}16 \\
121\end{array}$ & $\begin{array}{l}(11.7 \%) \\
(88.3 \%)\end{array}$ & $<0.0001$ \\
\hline
\end{tabular}

The proportion of subjects who made at-fault safety errors with either cut off value $(0 v \geqslant 1$ or $0 v \geqslant 2)$ was higher in the Alzheimer's disease group (Fisher's exact test). There was no statistically significant difference in the ages or MMSE scores between the "safe" and "unsafe" Alzheimer subgroups (Wilcoxon rank sum test).

$A D$, Alzheimer's disease; MMSE, mini-mental state examination; $y$, years. 
Inability to recognise traffic signs ${ }^{23}$ and landmarks may be an early complication in patients with Alzheimer's disease. $^{24}{ }^{25}$ Although multiple studies have evaluated on-theroad driving in Alzheimer subjects, ${ }^{86-32}$ to our knowledge, the current study is the first to test landmark and traffic sign identification during actual driving. Although basic visual sensory functions such as CS, FVA, and NVA correlated with LTIT performance, visual perception alone is unlikely to explain the difficulties in LTIT because the group differences remained significant after statistical correction for these measures, and visual acuity (FVA, NVA) was not a predictor by regression modelling. The finding that JLO and SFM predict landmark and traffic sign identification and driving safety, respectively, underscores the role of higher order visuoperceptual abilities, including motion processing, in visual search and object recognition during automobile driving. Our findings are consistent with reports that visual attention, ${ }^{67}$ reading, object localisation and recognition, ${ }^{11} 3334$ and visuospatial abilities ${ }^{7}$ are impaired even in early Alzheimer's disease. ${ }^{5-8} 10-12$

Regression modelling showed that the TMT-B is an independent predictor of landmark and traffic sign identification rate. TMT-B depends on cognitive flexibility to switch attention between two competing tasks (tracking numbers and letters) and is considered a task of executive function. ${ }^{15}$ In a similar vein, the LTIT requires executive control over attention to switch between the tasks of searching for landmarks and controlling the vehicle on the road. AVLTrecall performance reflects the integrity of anterograde verbal memory and can be considered an index of general Alzheimer's disease severity. ${ }^{35-38}$ Correlation of both verbal and non-verbal abilities with LTIT outcomes suggests that LTIT depends on distributed neural systems involving both hemispheres.

A subset of drivers with Alzheimer's disease did not make any safety errors, suggesting that some individuals with mild dementia remain fit drivers and may be allowed to continue to drive. ${ }^{39}$ The current findings indicate that the approach to assessments of driver fitness in at-risk drivers with cognitive impairment can benefit from introducing controlled challenges of vision, perception, memory, and attention during driving scenarios implemented in an instrumented vehicle. Future work in this area must address the relation between relatively high frequency, low severity safety errors such as we measured in ARGOS and low frequency, high severity incidents such as injurious crashes.

\section{ACKNOWLEDGEMENTS}

Supported by: NIA AG 17717 and NIA AG 15071.

\section{Authors' affiliations}

E Y Uc, S W Anderson, Division of Neuroergonomics, Department of Neurology, University of lowa Hospitals and Clinics, lowa City, lowa, USA

M Rizzo, Division of Neuroergonomics, Department of Neurology, University of lowa Hospitals and Clinics

Q Shi, J D Dawson, Department of Biostatistics, University of lowa Hospitals and Clinics

Competing interests: none declared

\section{REFERENCES}

1 Owsley C, McGwin G. Vision impairment and driving. Surv Ophthalmol 1999; 43:535-50.

2 Burg A. Vision and driving: a report on research. Hum Factors $1971 ; 13: 79-87$.

3 Mielke R, Kessler J, Fink G, et al. Dysfunction of visual cortex contributes to disturbed processing of visual information in Alzheimer's disease. Int J Neurosci 1995;82:1-9.

4 Morrison JH, Hof PR, Bouras C. An anatomic substrate for visual disconnection in Alzheimer's disease. Ann NY Acad Sci 1991;640:36-43.
5 Rizzo M, McGehee DV, Dawson JD, et al. Simulated car crashes at intersections in drivers with Alzheimer disease. Alzheimer Dis Assoc Disord 2001;15:10-20.

6 Rizzo M, Anderson SW, Dawson J, et al. Visual attention impairments in Alzheimer's disease. Neurology 2000;54:1954-9.

7 Rizzo M, Anderson SW, Dawson J, et al. Vision and cognition in Alzheimer's disease. Neuropsychologia 2000;38:1157-69.

8 Duchek JM, Hunt L, Ball K, et al. Attention and driving performance in Alzheimer's disease. J Gerontol B Psychol Sci Soc Sci 1998;53:130-41.

9 Rizzo M, Nawrot M. Perception of movement and shape in Alzheimer's disease. Brain 1998;121:2259-70.

10 Rizzo M, Reinach S, McGehee D, et al. Simulated car crashes and crash predictors in drivers with Alzheimer disease. Arch Neurol 1997;54:545-51.

11 Giannakopoulos P, Gold G, Duc M, et al. Neuroanatomic correlates of visual agnosia in Alzheimer's disease: a clinicopathologic study. Neurology 1999;52:71-7

12 Pantel J. Alzheimer's disease presenting as slowly progressive aphasia and slowly progressive visual agnosia: two early reports. Arch Neurol 1995:52:10.

13 McKhann G, Drachman D, Folstein M, et al. Clinical diagnosis of Alzheimer's disease: report of the NINCDS-ADRDA Work Group under the auspices of Department of Health and Human Services task force on Alzheimer's disease. Neurology 1984;34:939-44

14 Salmon DP, Thomas RG, Pay MM, et al. Alzheimer's disease can be accurately diagnosed in very mildly impaired individuals. Neurology 2002;59:1022-8

15 Lezak MD. Neuropsychological assessment, 3rd ed. New York: Oxford University Press, 1995

16 Ball KK. Real-world evaluation of visual function. Ophthalmol Clin North Am 2003; 16:289-98

17 Ball K, Owsley C, Sloane ME, et al. Visual attention problems as a predictor of vehicle crashes in older drivers. Invest Ophthalmol Vis Sci 1993:34:3110-23.

18 Owsley C, Ball K, Sloane ME, et al. Visual/cognitive correlates of vehicle accidents in older drivers. Psychol Aging 1991;6:403-15.

19 Pelli DG, Robson JG, Wilkins AJ. The design of a new letter chart for measuring contrast sensitivity. Vision Res 1988;2:187-99.

20 Ferris FL III, Kassoff A, Bresnick GH, et al. New visual acuity charts for clinical research. Am J Ophthalmol 1982;94:91-6.

21 Barry CJ, Smith D, Lennarson $P$, et al. The effect of wearing a restrictive neck brace on driver performance. Neurosurgery 2003;53:98-102

22 Rizzo M, McGehee D, Petersen $A D$, et al. Development of an unobtrusively instrumented field research vehicle for objective assessments of driving performance. In: Rothengatter T, Carbonnel VE, eds. Traffic and transport psychology: theory and application. New York: Pergamon Press, 1997:203-8

23 Carr DB, LaBarge E, Dunnigan K, et al. Differentiating drivers with dementia of the Alzheimer type from healthy older persons with a Traffic Sign Naming test. J Gerontol A Biol Sci Med Sci 1998;53:M135-9.

24 Aguirre GK, D'Esposito $M$. Topographical disorientation: a synthesis and taxonomy. Brain 1999;122:1613-28.

25 Cherrier MM, Mendez M, Perryman K. Route learning performance in Alzheimer disease patients. Neuropsychiatry Neuropsychol Behav Neurol 2001;14:159-68.

26 Duchek JM, Carr DB, Hunt L, et al. Longitudinal driving performance in earlystage dementia of the Alzheimer type. J Am Geriatr Soc 2003;51:1342-7.

27 Wild K, Cotrell V. Identifying driving impairment in Alzheimer disease: a comparison of self and observer reports versus driving evaluation. Alzheimer Dis Assoc Disord 2003;17:27-34.

28 Dobbs AR. Evaluating the driving competence of dementia patients. Alzheimer Dis Assoc Disord 1997;11(suppl 1):8-12.

29 Fox GK, Bowden SC, Bashford GM, et al. Alzheimer's disease and driving: prediction and assessment of driving performance. J Am Geriatr Soc 1997;45:949-53.

30 Hunt LA, Murphy CF, Carr D, et al. Reliability of the Washington University Road Test. A performance-based assessment for drivers with dementia of the Alzheimer type. Arch Neurol 1997:54:707-12.

31 Fitten LJ, Perryman KM, Wilkinson CJ, et al. Alzheimer and vascular dementias and driving. A prospective road and laboratory study. JAMA 1995;273:1360-5.

32 Hunt L, Morris JC, Edwards D, et al. Driving performance in persons with mild senile dementia of the Alzheimer type. J Am Geriatr Soc 1993:41:747-52.

33 Tetewsky SJ, Duffy CJ. Visual loss and getting lost in Alzheimer's disease. Neurology 1999;52:958-65

34 Kurylo DD, Corkin S, Rizzo M, et al. Greater relative impairment of object recognition than of visuospatial abilities in Alzheimer's disease. Neuropsychology 1996;10:74-81.

35 Estevez-Gonzalez A, Kulisevsky J, Boltes A, et al. Rey verbal learning test is a useful tool for differential diagnosis in the preclinical phase of Alzheimer's disease: comparison with mild cognitive impairment and normal aging. Int J Geriatr Psychiatry 2003;18:1021-8.

36 Tierney MC, Black SE, Szalai JP, et al. Recognition memory and verbal fluency differentiate probable Alzheimer disease from subcortical ischemic vascular dementia. Arch Neurol 2001;58:1654-9.

37 Gainotti G, Marra C, Villa G, et al. Sensitivity and specificity of some neuropsychological markers of Alzheimer dementia. Alzheimer Dis Assoc Disord 1998;12:152-62.

38 Tierney MC, Szalai JP, Snow WG, et al. Prediction of probable Alzheimer's disease in memory-impaired patients: a prospective longitudinal study. Neurology 1996;46:661-5.

39 Lundberg C, Johansson K, Ball K, et al. Dementia and driving: an attempt at consensus. Alzheimer Dis Assoc Disord 1997;11:28-37. 